\title{
Procrastination in Upper School Female Students at a New York City Private School Before and During the Coronavirus Pandemic of 2019
}

\author{
Chloe Padilla ${ }^{1}$ and Elaine Pan ${ }^{1}$ \\ ${ }^{1}$ The Chapin School, New York, New York, USA
}

\begin{abstract}
$\underline{\text { ABSTRACT }}$
Procrastination can affect some people in negative ways (Tice 1997). Teenagers are vulnerable to the detrimental effects of procrastination as they learn to manage school-life balance (Reinecke 2018). The coronavirus pandemic led to virtual education and less extracurricular activities which inadvertently increased unscheduled home time and possibly altering procrastination habits for teens. There is little research about the pandemic's effects on teenagers' education habits and the extent to which teenagers' procrastination habits have been affected. This study was conducted to help educators develop strategies to help teenagers manage school-life balance and decrease procrastination during the pandemic. This study reviewed the frequency of students' procrastination levels before and during the COVID-19 outbreak based on the "percentage of time passed when assignments were submitted between time, they were assigned at The Chapin School. 392 samples were collected and analyzed using the Chi-Square statistical test. The p-value of 0 was lower than the alpha of 0.01 , with a confidence level of $99 \%$. The alternative hypothesis that the level of procrastination in teenagers has decreased because of the coronavirus pandemic was accepted with $99 \%$ confidence. The collected data seemed to support the alternative hypothesis before analysis due to the differences in the frequency of the levels of procrastination before and during the pandemic. The results found in this study show educators that the new virtual platforms and schedules implemented during quarantine may have decreased the amount of procrastination in students.
\end{abstract}

\section{Introduction}

Procrastination is one of the major issues that affects the work ethic of teenagers and increases the amount of stress about the completion of a task (Reinecke 2018). Procrastination is defined as "to put off intentionally and habitually the doing of something that should be done" (Merriam-Webster Dictionary 2020). In order to decrease teenage procrastination, it is necessary to understand adolescent behavior, including the degree to which students are already procrastinating. The recent COVID-19 outbreak has affected the amount of free time that high school students have at home to do homework. This sudden increase of free time may have decreased the procrastination habits of teenagers who were previously engaged in this behavior on a regular basis.

The effect that this pandemic has had on these high school students can affect their ability to succeed through online schooling during these unusual times. The future of the world can be heavily dictated by the successful productivity of teenagers. School is one of the main parts of a child's life as they are developing (Roeser 2000). The teenage timeframe is crucial for learning new materials and about the world around them (Roeser 2000). Millions of teenagers around the world are quarantined, and their style of education has completely changed — for the better or worse. One of the main factors in a student's academic growth is the extent to which they procrastinate (Tice 1997). The habits of students' procrastination levels shifted because of the immense amount of stress that some teenagers are feeling due to this global pandemic. Some teenagers' lives have been shifted completely, and therefore their procrastination habits and quality of work shifted as well. The results of this study contribute valuable insight to ensure 
that those who will shape the future of the world are in the right academic state, meaning they have not fallen behind academically, after quarantine.

The sudden outbreak of the COVID-19 pandemic has forced many students into quarantine. Their educational system has been completely altered by the shift to online teaching. Most of the students' activities, such as clubs and keeping in contact physically with friends, have been cancelled to keep everyone safe. Therefore, this means that some students have nothing else urgent to do except for school. Staying at home provides students with the opportunity to pay more attention to their academics because they have fewer commitments than they may have had when school was in session. Even before the pandemic, procrastination was an issue that many students faced when completing assignments, especially projects that had a lengthy amount of time before the due date (Kachgal 2001). With virtual learning methods, students have more freedom during class. Students can mute their computers or turn off their video in order to bypass the possibility of teachers suspecting that the student is not focusing during the class period, despite the fact that teachers do still have some control over what the students see on their screens through the screen sharing option in Zoom. Also, because of the challenges that come with this pandemic, many schools have implemented new grading systems that encourage academic growth during this pandemic. For example, at The Chapin School, students cannot perform worse than they did in the previous two terms; however, some can improve their grades. This new grading system combined with the increased amount of unscheduled time has motivated students to procrastinate less during the pandemic. The sudden changes made for the benefit of students' mental health during the coronavirus pandemic may have encouraged students to procrastinate less.

The research specifically targets procrastination in and out of a pandemic period in high school students. The lifestyle adjustments made that have arisen during the coronavirus pandemic are new and unexpected. There is no published research about this topic specifically in the context of a pandemic. However, many studies specifically focus on measuring the levels of procrastination in young adults. Özer (2011) administered the Procrastination Assessment Scale-Student (PASS) test to measure the level of procrastination of high school, undergraduate, and graduate students. The PASS method was used in this study because the degree to which students believe they are procrastinating is important to assess the difference between students' perceived levels and actual levels of procrastination during the pandemic. The PASS test is a questionnaire that participants can complete in their homes and that only includes 44 questions. Kachgal (2001) also uses the PASS test to help students become aware of their own procrastination tendencies by having them self-report their levels of procrastination. The PASS test may not accurately assess the actual procrastination habits of students because students answer the questions based on their perception of their practices regarding academics. However, it is important to know how aware students are of their procrastination habits by comparing the data from the results of the data to their answers.

In Özer (2011), 53\% of the 79 high school students were procrastinators. Özer (2011) determined that the students who submitted the assignment after the median of the time given were procrastinators. However, in Kachgal (2001), 37.68\% of the undergraduate students tested were aware of their procrastination habits and believed they always or nearly always procrastinate.

Kachgal's research evaluated the procrastination cycles of young adults before the coronavirus pandemic. However, the global crisis caused by the coronavirus has deeply affected the education of students and may change the levels of procrastination in students. Because students have more free time at home than in a monitored school environment, their procrastination habits may have been affected. The previous research on this topic only focused on the subjects' self-awareness of their study habits did not evaluate how they have procrastinated through data. This research will evaluate procrastination study patterns by analyzing data from the times that teenage students actually performed a mandatory assignment before and during the pandemic.

\section{Materials and Methods}

This research specifically focused on the level of procrastination high school students partook in through comparing the differences of procrastination levels before and during a pandemic. In order to avoid biased data, the majority of 
the data was taken from mandatory forms electronically submitted by the upper school students at The Chapin School in New York City. Because the data of time submissions was collected by samples of convenience, the bias was still extremely high in this study. However, the selective demographic of upper school students at the single sex Chapin School served to accomplish the goal of this study to help teachers and administrators at the institution better understand the procrastination habits of their own students. Since information about the procrastination of Chapin students is extremely useful, the bias was acceptable.

This data is important to gauge how students are reacting to the sudden change in the school environment in terms of their deadline management. Another part of this study will ask the students how they feel regarding their habits of efficiency and whether those have been affected by having more "down time". It is critical that students are aware of the changes or consistencies of their working habits so that they can improve their academic efficiency.

The main goals of this project were to assess the extent to which high school students are procrastinating academically and how their studying habits were affected during a pandemic. The coronavirus pandemic has created new boundaries between students and teachers, physically and educationally. Many students have not had to travel and were unable to participate in extracurricular activities leading to many more hours at home of unscheduled time. This study compared the differences in procrastination habits for each participating student for two mandatory assignments before and during the quarantine to correctly determine how students have reacted to this pandemic through their academic work. The PASS test was administered so that the students' recognition of their working patterns was recorded during the pandemic. Students' perception regarding the intensity of their own procrastination was then compared with the time submission data from the high school. The independent variable was the time of the submission (before/after coronavirus outbreak affected schools). The dependent variable was the level of procrastination of the students (Figure 1). The $\mathrm{H}_{0}$ was the level of procrastination in teenagers has stayed the same as a result of the coronavirus pandemic. The $\mathrm{H}_{\mathrm{a}}$ was the level of procrastination in teenagers has decreased as a result of the coronavirus pandemic. The only materials that were necessary for this project is the poster and the google forms with the time submissions.

\section{Results}

The results of the time submissions were analyzed with the Chi-squared statistical analysis test. The method in which the data was analyzed is that the closer that a student hands in the assignment before the deadline, the more they have procrastinated (Figure 1).

Table 1. Frequency of Student's Procrastination Levels Before and During the COVID-19 Outbreak

\begin{tabular}{|c|c|c|c|c|c|c|}
\hline & None & Slight & Moderate & $\begin{array}{c}\text { Moderately } \\
\text { severe }\end{array}$ & Severe & Total \\
\hline Before & 40 & 19 & 7 & 103 & 27 & 196 \\
\hline During & 134 & 11 & 9 & 32 & 10 & 196 \\
\hline Total & 174 & 30 & 16 & 135 & 37 & \\
\hline
\end{tabular}

These percentages were chosen to convey the level of the students' procrastination levels. Unlike in Özer (2011), which only used $50 \%$ as the barrier for procrastination, more specification is needed when determining the procrastination level of students. Students who handed in their assignments as soon as they received them, before $50 \%$, did not procrastinate at all, because they successfully managed their time. Students that submitted from $50 \%$ to $69 \%$ procrastinated a miniscule amount - the slight amount of procrastination exhibited most likely has little to no reflection on the quality of the assignment. Students that submitted the assignment from $70 \%$ to $84 \%$ procrastinated a moderate amount because they were close to the deadline. Moderate procrastination is highly likely if a student forgot 
about an assignment and submitted it as soon as they found out about it. Students that submitted the assignment from $85 \%$ to $100 \%$ have moderately severe procrastination because they most likely started or finished the assignment right before the deadline. This type of procrastination may have become detrimental to the quality of the work submitted by this student. If an assignment was handed in after the due date, then the students have severely procrastinated to the point at which it heavily impacts their grade on the assignment.

All the students were initially informed of the deadline and contents of the mandatory assignment or form at the same time through an email. Another form anonymously asked the upper school students to complete the PASS test.

The Chi-Square test yielded a p-value of 0 . An alpha of 0.01 and a confidence level of $99 \%$ was chosen. The largest frequency before the coronavirus pandemic (103) was in the moderately severe category (Table 2). The smallest frequency before the coronavirus pandemic (7) was in the moderate category. The largest frequency during the coronavirus pandemic (134) was in the no procrastination (none) category. The smallest frequency during the coronavirus pandemic (9) was in the moderate category.

Table 2: Percentage of Time Passed between Assignment and Due Date of Any Assignment with Matching Designated Level of Procrastination

\begin{tabular}{|l|l|}
\hline $\begin{array}{l}\text { Percentage of Time Passed when Submitted } \\
\text { Between Time Assigned and Time Due }\end{array}$ & Level of Procrastination \\
\hline $50 \%$ & Slight procrastination \\
\hline $70 \%$ & Moderate procrastination \\
\hline $85 \%$ & Moderately severe procrastination \\
\hline Completed after due date & Severe procrastination \\
\hline
\end{tabular}

The alternative hypothesis stating that the level of procrastination in teenagers has decreased as a result of the coronavirus pandemic was accepted because the p-value of 0 was significantly less than the alpha of 0.01 . The collected data seemed to support the alternative hypothesis before analysis because of the drastic differences between the number of students that procrastinated before and during the pandemic (Table 1). Significantly fewer students procrastinated during the pandemic than before the pandemic. As a result of the increased amount of unscheduled time that students have the data supported that students likely had more time to complete their work and procrastinate less. Therefore, the number of students with any procrastination habits decreased during this pandemic.

\section{Discussion}

This study focused on the growing issue of academic procrastination, especially in high school students. This research study revealed that procrastination in upper school female students at a New York City private institution decreased during the coronavirus pandemic of 2019. The increase in free time likely facilitated the student's ability to complete their work in a timelier fashion with less time pressure from extracurricular activities and travel to and from school. There may have been concerns that environmental stress and remote learning with increased home time may have led to decreased productivity in teenage students, however the decreased procrastination seen in students suggests otherwise.

Some teenagers may believe that they do not have problems with managing their time. However, the data tells a different story, as a significant number of students wait until the last minute to finish their tasks. Procrastination can become a habit that may decrease the quality of work that one completes (Tice 1997). Time management is a critical life skill that is necessary for anyone who wants to be successful and seen as a professional.

This study was conducted to help educators develop strategies to help teenagers manage school-life balance and risk of procrastination during the pandemic. If this quarantine continues through the next school year, teachers 
will need to continue to adjust their teaching methods to ensure that all their students are engaging fully and monitoring their procrastination habits while in quarantine. The extent that students' habits of procrastination has changed because of the pandemic is essential for teachers to be aware so that they can help their students remain or become focused academically. The results of this study will be shared with administration and faculty so that any necessary changes can take place to facilitate strategies for procrastination can return to minimize the threat of procrastination that students are facing.

In the future, studies can be conducted to target a broader audience, such as male and female students from different public and private schools. Also, the importance and interschool differences can be evaluated to determine if procrastination is more of an issue with students who do not have the drive to succeed academically. The following alternative hypothesis could be tested: the level of procrastination in students who do not desire to succeed in school is increased compared to academically motivated students.

\section{Acknowledgment}

I would like to thank Ms. Elaine Pan for helping me with this project.

\section{References}

Kachgal MM, Hansen LS, Nutter KJ. 2001. Academic Procrastination Prevention/Intervention: Strategies and Recommendations. Journal of Developmental Education. [accessed 2020 Abr 29]; 25(1): 14-21. https://search.proquest.com/docview/228526473/fulltextPDF/FD9F8DC3BA9444E7PQ/1 ?accountid=35791.

Kim J, et al. 2017 Apr 10. Effects of time perspective and self-control on procrastination and Internet addiction. Journal of Behavioral Addictions. [accessed 2020 Apr 29]; 6(2), 229-236. file://Users/2022chloep/Downloads/[20635303\%20\%20Journal\%20of\%20Behavioral\%20Addictions]\%20Effects\%20of\%20time\%20perspective $\% 20$ and $\% 20$ selfcontrol $\% 20$ on $\% 20$ procrastination $\% 20$ and $\% 20$ Internet $\% 20$ addiction.pdf.

Merriam-Webster Dictionary. 2020. "Procrastinate.” Merriam-Webster.com Dictionary. [accessed 2020 May 11]. https://www.merriam-webster.com/dictionary/procrastinate. Accessed 11 May. 2020.

Mortazavi F, Mortazavi SS, Khosrorad R. 2015 Sep 24. Psychometric Properties of the Procrastination Assessment Scale-Student (PASS) in a Student Sample of Sabzevar University of Medical Sciences. Iran Red Crescent Med J. [accessed 2020 Apr 29]; 17(9): e28328. http://ircmj.com/articles/16707.html.

Özer BU. 2011. A Cross Sectional Study on Procrastination: Who Procrastinate More?. International Proceedings of Economics Development and Research. [accessed 2020 Abr 29]; 18. http://www.ipedr.com/vol18/8-ICERI2011-R00015.pdf.

Reinecke L, et al. 2018 June 11. The Relationship Between Trait Procrastination, Internet Use, and Psychological Functioning: Results From a Community Sample of German Adolescents. Frontiers in Psychology. [accessed 2020 Apr 29]; 9: 913. https://doi.org/10.3389/fpsyg.2018.00913.

Roeser RW, Eccles JS, Sameroff AJ. 2000 May. School as a Context of Early Adolescents' 
Academic and Social-Emotional Development: A Summary of Research Findings. The Elementary School Journal. [accessed 2020 May 13]; 100(5): 443-471.

file://Users/2022chloep/Downloads/RoeserEcclesSameroff2000AdolescenceSchooling.pdf.

Solomon LJ, Rothblum ED. 1994. Procrastination Assessment Scale-Students (PASS).

Measures for Clinical Practice. [accessed 2020 Apr 29]; 2: 446-452.

https://iiw.kuleuven.be/english/research/readystemgo/archief/intern/WP1/Action-2/intervention-

tools/instruments/Procrastination_Assessment_Scale\%20-PASS.pdf.

Tice DM, Baumeister RF. 1997 Nov 1. Longitudinal Study of Procrastination, Performance,

Stress, and Health: The Costs and Benefits of Dawdling. Case Western Reserve University. [accessed 2020 May 12]; 8(6): 454-458. https://citeseerx.ist.psu.edu/viewdoc/download?doi=10.1.1.461.1149\&rep=rep1\&type=pdf. 